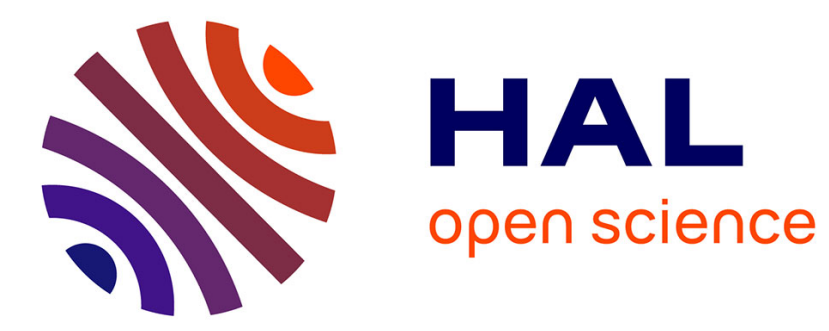

\title{
Contribution of the terahertz vibrations to the high temperature thermal conductivity of vitreous silica
}

Giacomo Baldi, Elisa Fabiani, Aldo Fontana, Valentina Giordano, Giulio Monaco, Giancarlo Ruocco, Francesco Sette

\section{- To cite this version:}

Giacomo Baldi, Elisa Fabiani, Aldo Fontana, Valentina Giordano, Giulio Monaco, et al.. Contribution of the terahertz vibrations to the high temperature thermal conductivity of vitreous silica. Philosophical Magazine, 2008, 88 (33-35), pp.3915-3923. 10.1080/14786430802438192 . hal-00513965

\section{HAL Id: hal-00513965 \\ https://hal.science/hal-00513965}

Submitted on 1 Sep 2010

HAL is a multi-disciplinary open access archive for the deposit and dissemination of scientific research documents, whether they are published or not. The documents may come from teaching and research institutions in France or abroad, or from public or private research centers.
L'archive ouverte pluridisciplinaire HAL, est destinée au dépôt et à la diffusion de documents scientifiques de niveau recherche, publiés ou non, émanant des établissements d'enseignement et de recherche français ou étrangers, des laboratoires publics ou privés. 


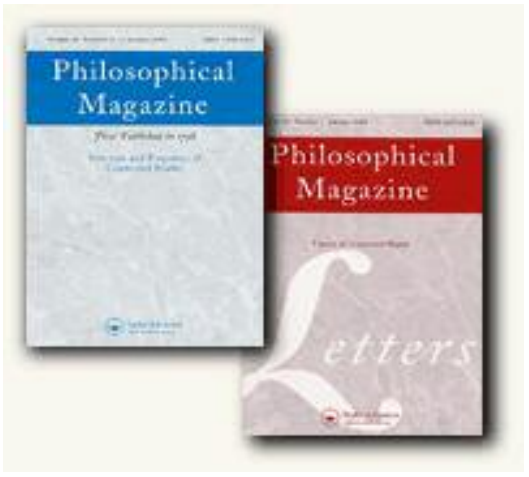

\section{Contribution of the terahertz vibrations to the high temperature thermal conductivity of vitreous silica}

\begin{tabular}{|r|l|}
\hline Journal: & Philosophical Magazine \& Philosophical Magazine Letters \\
\hline Mournal Selection: & Philosophical Magazine \\
\hline Author: & TPHM-08-May-0187.R1 \\
\hline Complete List of Authors: & $\begin{array}{l}\text { Baldi, Giacomo; INFM-CNR CRS-SOFT Operative Group in Grenoble } \\
\text { Fabiani, Elisa; CEA LETI Minatec, Departement pour la biologie et la } \\
\text { sante } \\
\text { Fontana, Aldo; University of trento, Physics; INFM-CNR CRS-SOFT } \\
\text { Giordano, Valentina; European Synchrotron Radiation Facility } \\
\text { Monaco, Giulio; European Synchrotron Radiation Facility } \\
\text { Ruocco, Giancarlo; Univ. of Rome, Physics; INFM-CNR CRS-SOFT } \\
\text { Sette, Francesco; European Synchrotron Radiation Facility }\end{array}$ \\
\hline Keywords: & $\begin{array}{l}\text { amorphous solids, boson peak, neutron scattering, thermal } \\
\text { transport, X-ray scattering }\end{array}$ \\
\hline Keywords (user supplied): & amorphous solids, boson peak, neutron scattering \\
\hline \multicolumn{2}{|l}{$\begin{array}{l}\text { Note: The following files were submitted by the author for peer review, but cannot be converted } \\
\text { to PDF. You must view these files (e.g. movies) online. }\end{array}$} \\
\hline articleSio2.tex
\end{tabular}

\section{ScholarONE" \\ Manuscript Central}




\section{Contribution of the terahertz vibrations to the high temperature thermal conductivity of vitreous silica}

\begin{tabular}{|r|l|}
\hline Journal: & Philosophical Magazine \& Philosophical Magazine Letters \\
\hline Manuscript ID: & TPHM-08-May-0187.R1 \\
\hline Journal Selection: & Philosophical Magazine \\
\hline Author: & $29-J u l-2008$ \\
\hline Complete List of Authors: & $\begin{array}{l}\text { Baldi, Giacomo; INFM-CNR CRS-SOFT Operative Group in Grenoble } \\
\text { Fabiani, Elisa; CEA LETI Minatec, Departement pour la biologie et la } \\
\text { sante } \\
\text { Fontana, Aldo; University of trento, Physics; INFM-CNR CRS-SOFT } \\
\text { Giordano, Valentina; European Synchrotron Radiation Facility } \\
\text { Monaco, Giulio; European Synchrotron Radiation Facility } \\
\text { Ruocco, Giancarlo; Univ. of Rome,, Physics; INFM-CNR CRS-SOFT } \\
\text { Sette, Francesco; European Synchrotron Radiation Facility }\end{array}$ \\
\hline Keywords: & $\begin{array}{l}\text { amorphous solids, boson peak, neutron scattering, thermal } \\
\text { transport, X-ray scattering }\end{array}$ \\
\hline Keywords (user supplied): & amorphous solids, boson peak, neutron scattering \\
\hline & \multicolumn{2}{|l}{} \\
\hline
\end{tabular}

\section{S ScholaroNE \\ Manuscript Central}




\title{
Contribution of the terahertz vibrations to the high temperature thermal conductivity of vitreous silica
}

\author{
G. Baldi ${ }^{\dagger *}$, E. Fabiani $i^{\ddagger}$, A. Fontana ${ }^{£}$, V. Giordano" \\ G. Monaco"l, G. Ruocco ${ }^{\dagger \dagger}$ and F. Settel \\ ${ }^{\dagger}$ INFM-CNR CRS-SOFT OGG c/o E.S.R.F., BP220, F-38043 Grenoble Cedex, France; \\ ${ }^{\ddagger}$ CEA LETI Minatec, Grenoble Cedex 9, France \\ $\S$ Physics Department, Trento University, Via Sommarive 14, 38050 Povo, Trento, Italy \\ "INFM-CNR CRS-SOFT, c/o Roma University "La Sapienza", 00185, Roma, Italy \\ "European Synchrotron Radiation Facility, BP220, F-38043 Grenoble Cedex, France \\ ${ }^{\dagger}$ Physics Department, Roma University "La Sapienza", 00185, Roma, Italy
}

(Received 00 Month 200x; final version received 00 Month 200x)

\begin{abstract}
A combined inelastic X-rays and neutron scattering experiment is performed on the prototypical glass vitreous silica. The complementarity of the two techniques is exploited to determine the dynamic structure factor in a wide range of energies and wave-vectors. The vibrational modes spectral density is then used to compute the high temperature thermal conductivity. The acoustic-like modes persisting at $\mathrm{THz}$ frequencies are shown to represent a relevant heat conduction channel, although they cannot account for the entire thermal conductivity.
\end{abstract}

Keywords: boson peak, thermal transport, amorphous solids, X-ray scattering, neutron scattering

\section{Introduction}

The peculiar temperature dependence of the thermal conductivity of glasses has been the subject of many investigations both from the experimental [1-10] and the theoretical side [11-15]. In dielectric glasses at low temperatures heat is carried by acoustic excitations, whose mean free path can be determined by means of spectroscopic [16-18] or acoustic [19-21] measurements. Three temperature ranges can be identified, where different sound damping mechanisms dominate the sound attenuation process. In the low temperature range, for $T<1 \mathrm{~K}$, the thermal conductivity is quadratic in temperature and the sound damping is governed by tunneling two level systems [22]. For temperatures around $10 \mathrm{~K}$ the conductivity presents a plateau, whose nature has been strongly debated in the literature $[2,4-6]$ and is still an open issue $[9,10,12,15]$. Then, around $30 \mathrm{~K}$, the thermal conductivity rises again above the plateau $[1,7]$.

Previous estimates $[1,3,5-7,23]$ of the thermal conductivity of glasses where limited by the knowledge of the acoustic mean free path only for relatively low frequencies, below the 10-50 GHz range explored by Brillouin light scattering [16]. More recently the determination of the acoustic mean free path of amorphous thin films for frequencies up to $400 \mathrm{GHz}$ by means of the picoseconds optical

${ }^{*}$ Corresponding author. Email: giacomo.baldi@esrf.fr 
technique [21] was used as a base for an estimate of the thermal conductivity above the plateau [8]. In all these works the mean free path, $l(\omega)$, in the $\mathrm{THz}$ range was assumed to be either constant $[1,6]$ as a function of frequency or proportional to the wavelength $[5,7,23]$. The inelastic X-rays scattering technique has allowed to detect acoustic-like excitations showing a linear dispersion relation up to frequencies of the order of some $\mathrm{THz}$, allowing for a direct determination of the high frequency mean free path.

We present in this paper a combined inelastic X-rays (IXS) and neutron (INS) scattering investigation of the vibrations of the prototypical glass vitreous silica. These two techniques allow to probe the vibrational dynamics of glasses for frequencies in the $\mathrm{THz}$ range and exchanged wave-vectors $q$ above $1 \mathrm{~nm}^{-1}$. The experimentally determined dynamic structure factor $S(q, \omega)$ is used to estimate the contribution of the $\mathrm{THz}$ vibrations to the thermal conductivity above the plateau. The involved temperatures are, in fact, in the range above $30 \mathrm{~K}$, corresponding, in the dominant phonon approximation, to the frequency window at present accessible to IXS and INS.

\section{Experiment}

The IXS experiment was carried out at the inelastic X-rays scattering beam line ID16 at the European Synchrotron Radiation Facility in Grenoble, France. To optimize the flux intensity on the sample we used the $\operatorname{Si}(9,9,9)$ reflection of the silicon crystal monochromator, giving an energy resolution of $2.8 \mathrm{meV}$. The IXS spectra have been collected at fixed exchanged wave-vector values between 4 and $15 \mathrm{~nm}^{-1}$ as a function of the exchanged energy. Scans at the two temperatures $T=1570 \mathrm{~K}$ and $T=920 \mathrm{~K}$ were performed in order to span a wide temperature range above and below the glass transition $\left(T_{g} \sim 1450 \mathrm{~K}\right)$ and to enhance the inelastic spectral features. The Suprasil fused quartz sample of $1.4 \mathrm{~mm}$ thickness and $2 \mathrm{~mm}$ diameter was radiatively heated by a graphite foil in a vacuum chamber.

The neutron inelastic spectra were collected at the neutrons time of flight spectrometers IN4 and IN6 at the Institut Laue-Langevin in Grenoble. The cold neutron spectrometer IN6 has been used to span the wave-vector region between 8 and 25 $\mathrm{nm}^{-1}$, which partially overlaps to the one explored by IXS, with a high energy resolution of around $0.15 \mathrm{meV}$ but with the drawback of a limited dynamical range. The measurements were performed with an incoming neutrons wavelength of $4.12 \AA$. The IN4 thermal neutron spectrometer was employed to span the high exchanged wave-vector region $q>15 \mathrm{~nm}^{-1}$. The chosen incoming neutron wavelength of 1.53 $\AA$ gave an energy resolution of $1.5 \mathrm{meV}$. The signal from a Suprasil v- $\mathrm{SiO}_{2}$ disk of 5 $\mathrm{cm}$ diameter and $4 \mathrm{~mm}$ width was measured at the three temperatures $\mathrm{T}=15 \mathrm{~K}, 50$ $\mathrm{K}$ and $300 \mathrm{~K}$. Further details on the experiments will be published in a forthcoming article [24].

A selection of spectra obtained with the three spectrometers is presented in figure 1. Here the inelastic part of the dynamic structure factor is plotted as a function of energy at fixed $q$ values. The elastic line, whose shape is given by the resolution function, was subtracted from the spectra after having determined its intensity by means of a chi-square minimization algorithm. The IXS spectra are characterized by the presence of two inelastic peaks, confirming the observations of a previous work [25]. The inelastic part of $S(q, \omega)$ is thus modeled to a two components function. The model function consists of the sum of a log-normal function to describe the lower frequency peak plus a damped harmonic oscillator (DHO) for the higher frequency one. 

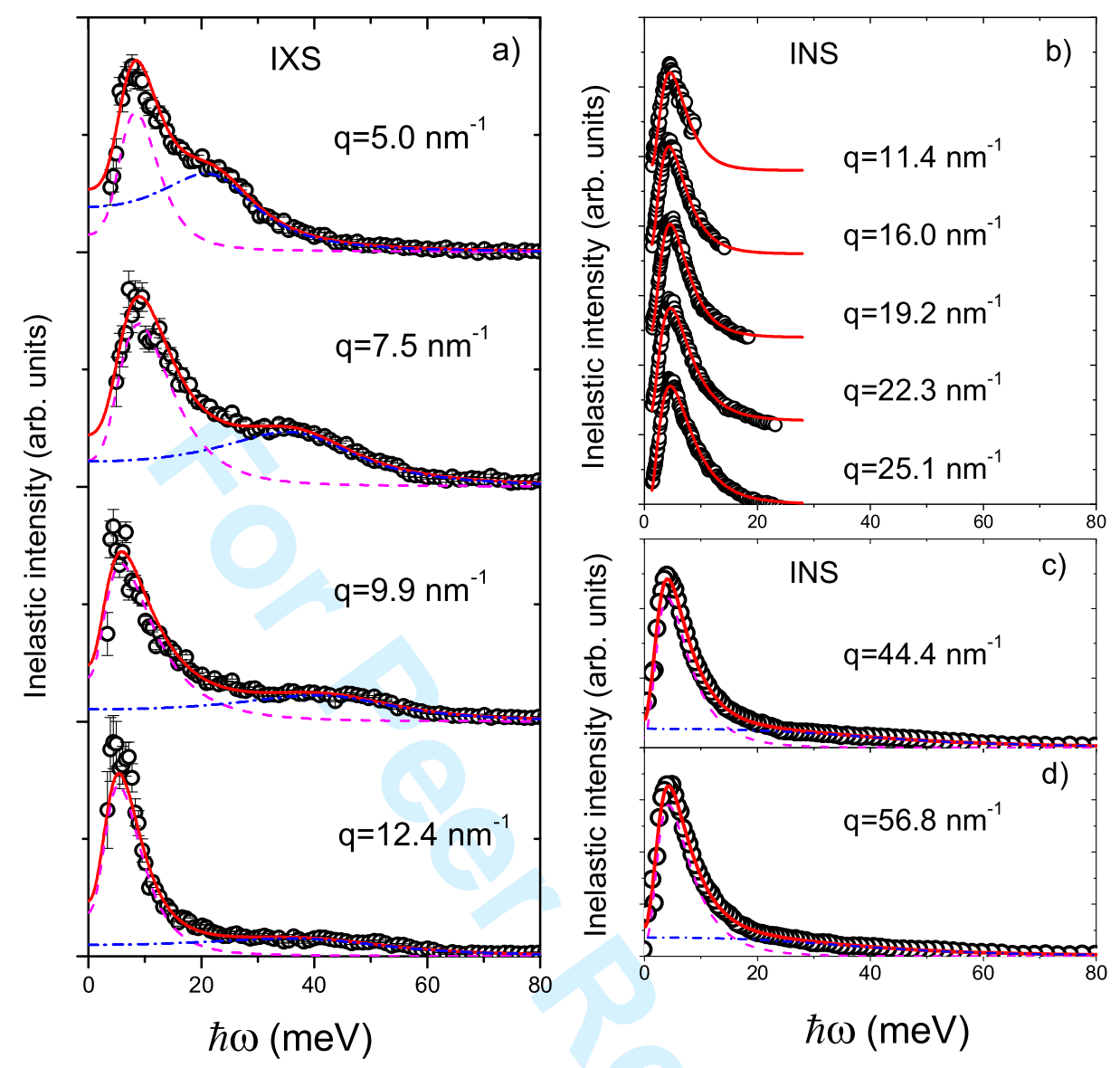

Figure 1. (Color online). Inelastic part of the IXS and INS spectra at selected exchanged wave-vectors. The inelastic intensity is computed as $S_{\text {inel }}(q, \omega)$ divided by $\hbar \omega[n(\omega)+1] /\left(k_{B} T\right)$, where $n(\omega)$ is the Bose population factor and $k_{B}$ the Boltzmann constant. The best fitting function is plotted as a continuous (red) line, the two inelastic components as dashed (magenta) and dash-dotted (blue) lines. The ordinate axis is in arbitrary units and the peak intensities within each panel are normalized to the lower frequency peak. The spectra are displaced of a constant amount one with respect to the other. Panel a): IXS spectra at $\mathrm{T}=1570 \mathrm{~K}$; b): INS spectra measured with the IN6 spectrometer at $\mathrm{T}=300 \mathrm{~K}$; c)-d): INS spectra measured with the IN4 spectrometer at T=300 K.

The positions $\hbar \Omega$ of the peaks appearing in the IXS and INS current correlation function are plotted as a function of $q$ in figure 2. The new data sets (colored online) are here compared to previous IXS results (triangles and circles). In the low $q$ range $\left(q<4 \mathrm{~nm}^{-1}\right)$ a single excitation, which disperses at the macroscopic longitudinal speed of sound, is observed by means of IXS. At higher wave-vectors two vibrational branches appear in the spectra.

The longitudinal branch is marked by a positive dispersion, which is temperature independent in the explored temperature range. The positive dispersion sets in at 4 $\mathrm{nm}^{-1}$ as can be seen in the inset of figure 2, where the longitudinal mode dispersion curve measured with IXS is highlighted. The lower frequency component appearing in the IXS spectra is, on the contrary, non dispersive and located around the Boson Peak position. This feature is resolved with an higher contrast in the INS spectra as shown in figure 1. The higher frequency component appears in the INS spectra only for $q>30 \mathrm{~nm}^{-1}$, because it lies outside the experimental dynamical range at smaller wave-vectors. In the high $q$ range the two vibrational branches 


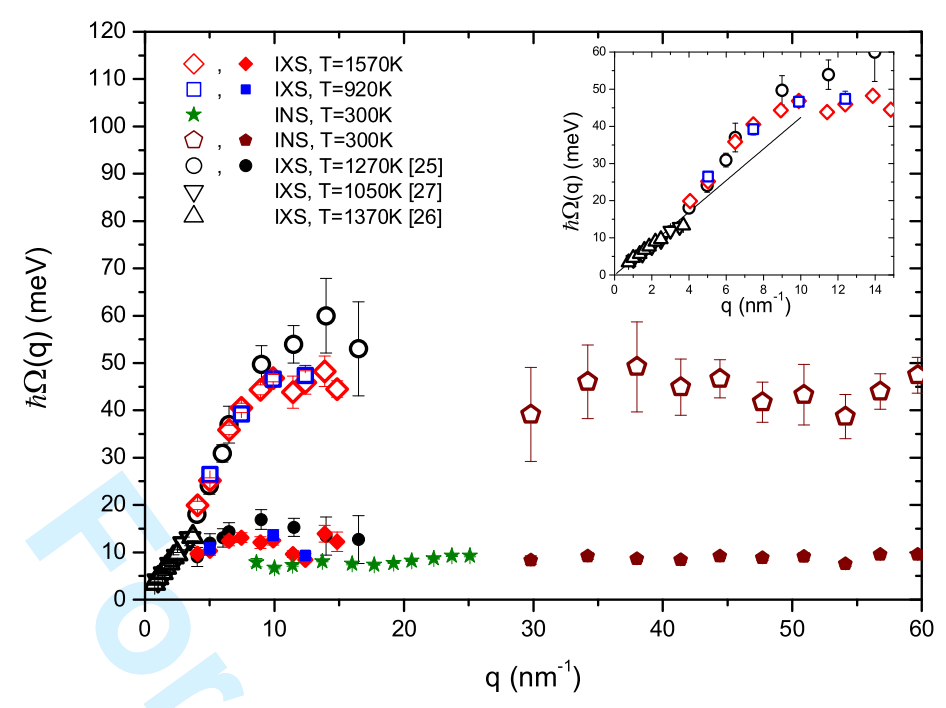

Figure 2. Dispersion relation for $\mathrm{v}-\mathrm{SiO}_{2}$. Open (longitudinal branch) and full (lower frequency component) diamonds: present work, $T=1570 \mathrm{~K}$ (red); open and full squares: present work, $T=920 \mathrm{~K}$ (blue); open and full circles: Ruzicka et al. [25], $T=1270 \mathrm{~K}$; upwards triangles: Scopigno et al., $T=1375$ $\mathrm{K}$ [26]; downwards triangles: Benassi et al., $T=1050 \mathrm{~K}$ [27]. The position of the peaks in the INS spectra

is reported as full stars (green) (IN6 data) and as open and full pentagons (IN4 data). The inset

highlights the longitudinal dispersion curve in the low- $q$ region as determined by IXS. The full line corresponds to the linear dispersion at the macroscopic longitudinal sound velocity.

are essentially non dispersive, with only small oscillations of the higher frequency component. The dynamic structure factor in this high $q$ region is converging to an effective density of vibrational states, $g(\omega)$. The two branches are in fact located at 10 and $50 \mathrm{meV}$, where peaks in $g(\omega)$ are observed in neutron experiments [28, 29] and in ab-initio numerical simulations [30].

\section{Thermal conductivity in the Kubo formulation}

The thermal Kubo formula relates the thermal conductivity to the correlation function of the heat current operator $\hat{S}(t)$. A detailed derivation of the relevant expressions can be found in a review by Allen and Feldmann [13]. Here we will simply recall the main results that allow a computation of the thermal conductivity from the experimental data. In the harmonic approximation the thermal conductivity can be expressed in terms of the mode diffusivity $D(\omega)$ as:

$$
k(T)=\frac{3 N}{V} \int_{0}^{\infty} d \omega C(\omega / T) g(\omega) D(\omega)
$$

where $C(\omega / T)=k_{B} x^{2} e^{x} /\left(e^{x}-1\right)^{2}$, with $x=\hbar \omega /\left(k_{B} T\right)$, is the specific heat of an oscillator of energy $\hbar \omega$ and the vibrational density of states $g(\omega)$ is normalized to unity. The mode diffusivity is function of the heat current operator $\hat{S}$ and of the resolvent $\hat{G}(\omega)=\left(\omega^{2}-\hat{M}\right)^{-1}$ of the dynamical matrix $\hat{M}[11,14]$ :

$$
D(\omega)=\frac{4}{3 \hbar^{2} \pi g(\omega)} \operatorname{Tr}\{\hat{S} \cdot \operatorname{Im}[\hat{G}(\omega)] \cdot \hat{S} \cdot \operatorname{Im}[\hat{G}(\omega)]\}
$$


Here $\operatorname{Tr}$ denotes the trace of the operator enclosed in brackets. In a crystal with mass disorder Flicker and Leath [11] showed that, in the framework of the coherent potential approximation, the trace can be computed in the base of the wavevectors. The resulting mode diffusivity can be expressed in terms of the inelastic part $S_{\text {inel }}(q, \omega)$ of the dynamic structure factor as:

$$
D(\omega)=\frac{2 V \omega^{2}}{3 \pi N g(\omega)} \int d q\left\{\frac{S_{\text {inel }}(q, \omega)}{S(q)[1-f(q)]}\right\}^{2}
$$

where $S(q)$ is the static structure factor and $f(q)$ the non ergodicity parameter, determined as the ratio between the elastic and the total integrated intensity. The frequency integral of $S_{\text {inel }}(q, \omega)$ is $S(q)[1-f(q)]$, the inelastic fraction of $S(q)$.

The expression for the thermal conductivity resulting from eq.(1) and (2) represents an extension of the phonon gas model in the Peierls-Boltzmann theory [13]. This approach allows to take into account the broadening of the peak even when this is not negligible compared to the peak position. In the usual phonon gas approximation each mode is assigned a specific heat $C(\omega / T)$ and a mean free path $\ell(\omega)$ and the diffusivity is $D(\omega)=v \ell(\omega) / 3$. Equation (2) can be regarded as a generalization because the inelastic part of the dynamic structure factor is integrated in both directions in the $\omega-q$ plane. In particular the specific heat is integrated over that group of modes that correspond to the same wave-vector, as can be seen by changing the integration order in equations (1) and (2).

The assumption that the heat current operator is diagonal in the wave-vectors base is a strong approximation in the case of a structurally disordered glass. As discussed by Allen and Feldman [13] in a topologically disordered system the operator $\hat{S}$ has non negligible off-diagonal terms on the base of the eigenstates of the dynamical matrix. However the information that one can extract from the experiment is limited to the diagonal contribution of equation (2).

\section{Discussion}

The evaluation of the thermal conductivity requires the knowledge of the sound attenuation of both the longitudinal and the transverse acoustic branches. The experimental data for the transverse modes are, however, limited to the ultrasound $[19,22]$ and BLS [31] techniques. These data indicate that the internal friction parameter, defined as $Q^{-1}=\ell^{-1} \lambda / 2 \pi$, assumes roughly the same value for both the transverse and the longitudinal modes if taken at the same frequency. This result is confirmed by molecular dynamic simulations [32] also in the high frequency region, where the attenuation is dominated by the structural disorder. Recasted in terms of the mean free path it implies $\ell_{T} / \ell_{L}=v_{T} / v_{L}$, where $L$ and $T$ denote the longitudinal and the transverse branch and $v_{L}$ and $v_{T}$ their sound velocities. In the following evaluation we will thus assume this ratio to be constant at all frequencies. It is worth noting that the prediction of the soft potential model is in agreement with this assumption [9].

The diffusivity computed for the longitudinal mode is plotted in figure 3 as a continuous line. In this estimate the Debye approximation is used for the density of vibrational states and a DHO function to model $S_{\text {inel }}(q, \omega)$, with the parameters determined from the measured spectra. The diffusivity for the transverse mode is computed in the same way from eq. (2) assuming the transverse peak width at a given frequency to coincide with the longitudinal one.

In the present evaluation of $k(T)$ the integral appearing in eq. (1) is computed in 

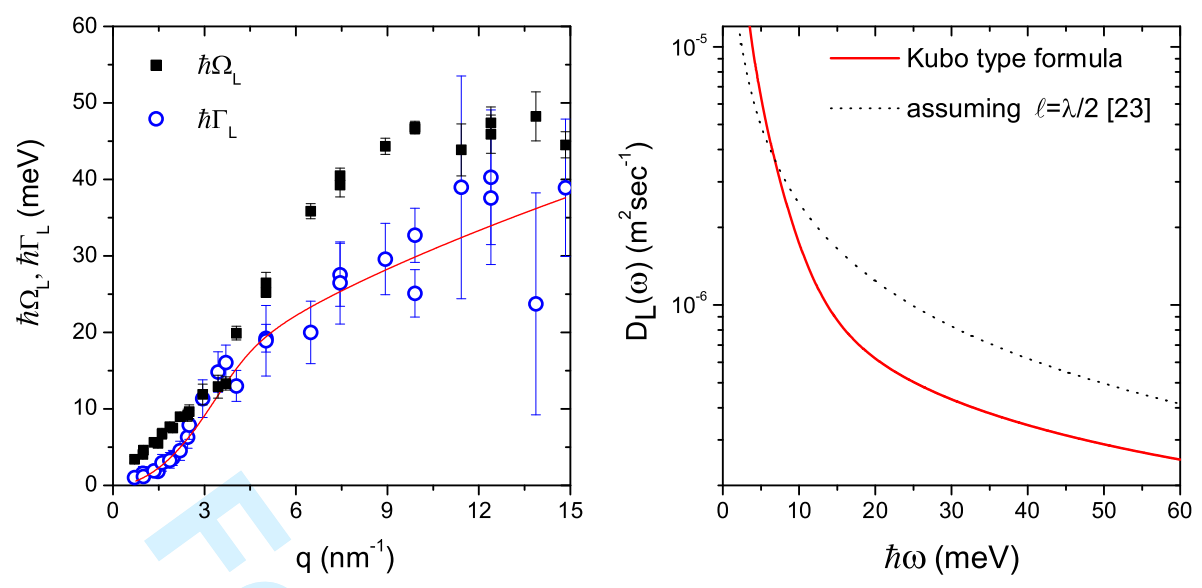

Figure 3. Left panel: wave-vector dependence of the peak position $\hbar \Omega$ (full squares) and peak width $\hbar \Gamma$ (open circles, blue) of the longitudinal component of the IXS spectra as determined from the DHO fit. The data for $q<4 \mathrm{~nm}^{-1}$ are taken from references $[26,27]$ as in figure 2. The line is a phenomenological function used to describe the $q$ dependence of $\Gamma$. Right panel: diffusivity of the longitudinal mode as a function of frequency. The continuous (red) line is the result for $D(\omega)$ from eq. (2). The value used in ref. [23] is included for comparison as a dotted line and corresponds to the choice $\ell=\lambda / 2$ in the phonon gas approach. (Color online).

the energy region spanned by the IXS technique, above $3 \mathrm{meV}$ in $\mathrm{v}-\mathrm{SiO}_{2}$ (see figure 2). This energy range corresponds to temperatures lying above the characteristic plateau appearing in the thermal conductivity in the 1-10 $\mathrm{K}$ temperature range. The actual nature of the plateau is source of debate in the literature $[9,10,15,18]$ and lies outside the scope of the present work.

The thermal conductivity obtained from equation (1) is plotted in figure 4 and compared to the measured one. A constant value, the dotted line in the figure, is added to the computed thermal conductivity to account for the small contribution of lower frequencies attenuation mechanisms which give rise to the plateau. This contribution will eventually decrease at high temperatures but the error associated to the assumption of a constant term is small, less than $10 \%$, with respect to the total $k(T)$. In the figure the prediction of the Kubo type formula of eq. (2) is compared to the one of the phonon gas model, in which the phonon mean free path is used to compute the diffusivity.

It is of some interest to compare the present result with a previous estimate by Cahill and Pohl [23]. They employed equation (1) in the Debye approximation using the usual phonon gas model result for the diffusivity. Applying concepts which date back to Einstein, they assumed the phonon mean free path to be equal to half the wavelength. Their result is in good agreement with the measured thermal conductivity. However the hypothesis of $\ell \sim \lambda / 2$ does not agree with the damping of the modes estimated from the IXS data. In this frequency range, in fact, the mean free path estimated from the width of the spectral density decreases as $\lambda^{2}$ up to $q$ around $4 \mathrm{~nm}^{-1}$ and then saturates at a value of the order of the interatomic distance, between $\lambda / 4$ and $\lambda / 6$. In terms of the diffusivity, the assumption of $\ell \sim$ $\lambda / 2$ in the phonon gas model gives the curve plotted as a dotted line in figure 3 . This diffusivity is clearly too high with respect to the one that can be extracted from the IXS dynamic structure factor.

Various assumptions at the base of the present thermal conductivity estimate need some further comment. A first strong assumption is the use of the Debye approximation for the density of states. This choice can be justified by the following 


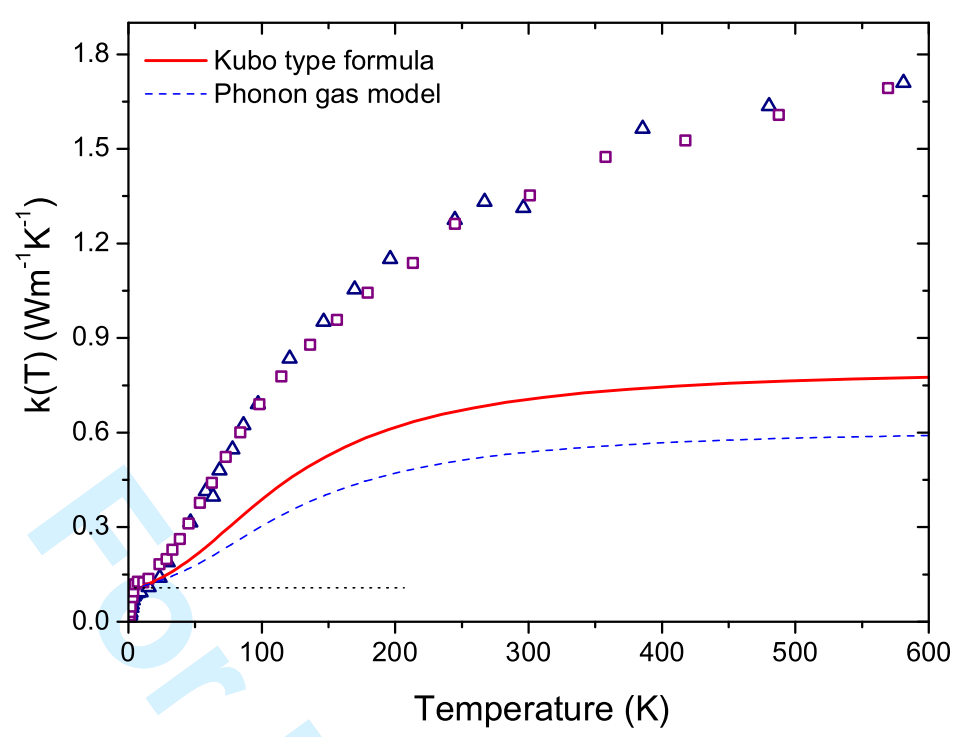

Figure 4. Thermal conductivity of vitreous silica. Squares (purple): from ref [23]; triangles (navy): from ref. [5]. The continuous line (red) is the estimate from equations (1) and (2). The phonon gas approximation is plotted for comparison as a dashed line (blue). The dotted line is the constant value added as an estimate of the plateau value and accounts for the omitted low energy, $\hbar \omega<3 \mathrm{meV}$, contributions. (Color online).

argument. If the total density of states is used in equation (1), the thermal conductivity is overestimated in the region of the plateau. This is due to the presence of the excess of states at the Boson Peak, which is located at a frequency around 1 $\mathrm{THz}$ and corresponds to the temperature region between 10 and $40 \mathrm{~K}$, thus in the upper part of the plateau. This modes are non dispersive, as shown in figure 2, and for this reason they cannot be included in the evaluation based on eq. (2), where only the diagonal elements of the heat current operator are used and a non-zero sound speed is required.

Probably the strongest assumption in the present evaluation is the omission of the off-diagonal contributions to the heat current operator. Unfortunately the off diagonal elements necessary for a complete determination of the heat current operator are not experimentally accessible. A deeper insight on the relation between vibrations and thermal conductivity would require a numerical simulation approach. The simulated spectra can then be checked against the experimental one, and the mode diffusivity computed from the knowledge of the eigenvectors and eigenfunctions of the dynamical matrix.

Furthermore, the simple harmonic approach here followed doesn't take into account any possible interaction between the acoustic-like modes and the cornerconnected tetrahedra librations. These modes appear to be relevant in vitreous silica [33, 34] and a more refined analysis should include also these anharmonic contributions. Such an analysis would however require more detailed spectroscopic data and further input from computer simulations.

\section{Conclusions}

We have presented an evaluation of the high temperature thermal conductivity of vitreous silica in the framework of the thermal Kubo equation. The diagonal 
contribution to the thermal conductivity has been computed from the experimentally determined dynamic structure factor. The combined used of IXS and INS techniques has allowed us to probe the vibrational dynamics in a wide range of exchanged energies and wave-vectors.

The acoustic-like excitations which persists in silica in the $\mathrm{THz}$ frequency range represent an important heat conduction channel, responsible for a relevant fraction of the total thermal conductivity. The remaining part of the concuctivity can be attributed to diffusons modes which participate to the heat conduction through the off-diagonal terms in the heat current operator as suggested by Allen and Feldman [13].

\section{Acknowledgments}

G. B. is indebted to prof. U. Buchenau for many fruitful discussions on the thermal conductivity in amorphous solids. C. Henriquet is kindly acknowledged for its support in the preparation of the high temperature set-up for the IXS experiment. We thank A. J. Dianoux for his help during the neutron experiments. E. F. acknowledges M. M. Koza for his support in the treatment of the neutrons data. Technical and financial support from the ILL facility is gratefully acknowledged.

\section{References}

[1] C. Kittel, Phys. Rev. 75 (1949) p.972.

[2] R. C. Zeller and R. O. Pohl, Phys. Rev. B 4 (1971) p.2029.

[3] G. A. Slack, in Solid State Physics, H. Ehrenreich, F. Seitz and D. Turnbull, eds., Academic Press, New York, 1979, Vol. 34, p.1.

[4] A. C. Anderson in Amorphous Solids, Low Temperature Properties, W. A. Phillips, ed., Springer, New York, 1981.

[5] J. J. Freeman and A. C. Anderson, Phys. Rev. B 34 (1986) p.5684; C. C. Yu and J. J. Freeman, ibid. 36 (1987) p.7620.

[6] J. E. Graebner, B. Golding and L. C. Allen, Phys. Rev B 34 (1986) p.5696.

[7] D. G. Cahill and R. O. Pohl, Phys. Rev. B 35 (1987) p. 4067.

[8] M. S. Love and A. C. Anderson, Phys. Rev. B 42 (1990) p.1845.

[9] U. Buchenau, Yu. M. Galperin, V. L. Gurevich, D. A. Parshin, M. A. Ramos and H. R. Schober, Phys. Rev. B 46 (1992) p.2798.

[10] R. Vacher, J. Pelous and E. Courtens, Phys. Rev. B 56 (1997) p.R481.

[11] J. K. Flicker and P. L. Leath, Phys. Rev. B 7 (1973) p.2296.

[12] P. Sheng and M. Zhou, Science 253 (1991) p.539.

[13] P. B. Allen and J. L. Feldman, Phys. Rev. B 48 (1993) p.12581.

[14] A. Alam and A. Mookerjee, Phys. Rev. B 72 (2005) p.214207.

[15] W. Schirmacher, Europhys. Lett. 73 (2006) p.892.

[16] A. S. Pine, Phys. Rev. 185 (1969) p.1187.

[17] P. Benassi, S. Caponi, R. Eramo, A. Fontana, A. Giugni, M. Nardone, M. Sampoli and G. Viliani, Phys. Rev. B 71 (2005) p.172201.

[18] C. Masciovecchio, G. Baldi, S. Caponi, L. Comez, S. Di Fonzo, D. Fioretto, A. Fontana, A. Gessini, S. C. Santucci, F. Sette, G. Viliani, P. Vilmercati and G. Ruocco, Phys. Rev. Lett. 97 (2006) p.035501.

[19] R. E. Strakna and H. T. Savage, J. Appl. Phys. 35 (1964) p.1445.

[20] W. Dietsche and H. Kinder, Phys. Rev. Lett. 43 (1979) p.1413.

$[21]$ T. C. Zhu, H. J. Maris and J. Tauc, Phys. Rev. B 44 (1991) p.4281.

$[22]$ B. Golding, J. E. Graebner and R. J. Schutz, Phys. Rev. B 14 (1976) p.1660.

[23] D. G. Cahill and R. O. Pohl, Ann. Rev. Phys. Chem. 39 (1988) p.93; Solid State Comm. 70 (1989) p.927; D. G. Cahill, S. K. Watson and R. O. Pohl, Phys. Rev. B 46 (1992) p.6131.

[24] G. Baldi, V. Giordano, G. Monaco, F. Sette, E. Fabiani, A. Fontana and G. Ruocco, Phys. Rev. B 77 (2008) p.214309.

[25] B. Ruzicka, T. Scopigno, S. Caponi, A. Fontana, O. Pilla, P. Giura, G. Monaco, E. Pontecorvo, G. Ruocco and F. Sette, Phys. Rev. B 69 (2004) p.100201(R).

[26] T. Scopigno, thesis, L'Aquila University, 1998; C. Masciovecchio, V. Mazzacurati, G. Monaco, G. Ruocco, T. Scopigno, F. Sette, P. Benassi, A. Cunsolo, A. Fontana, M. Krisch, A. Mermet, M. Montagna, F. Rossi, M. Sampoli, G. Signorelli and R. Verbeni, Philos. Mag. B 79 (1999) p.2013.

[27] P. Benassi, M. Krisch, C. Masciovecchio, V. Mazzacurati, G. Monaco, G. Ruocco, F. Sette and R. Verbeni, Phys. Rev. Lett. 77 (1996) p.3835.

[28] J. M. Carpenter and D. L. Price, Phys. Rev. Lett. 54 (1985) p.441.

[29] M. Arai, A. C. Hannon, A. D. Taylor, A. C. Wright, R. N. Sinclair and D. L. Price, Physica B 180 
(1992) p.779; M. Arai, A. C. Hannon, T. Otomo, A. Hiramatsu and T. Nishijima, J. Non Cryst. Solids $192 \& 193$ (1995) p.230.

[30] L. Giacomazzi and A. Pasquarello, J. Phys.: Condens. Matter 19 (2007) p.415112.

[31] R. Vacher, J. Pelous, F. Plique and A. Zarembowitch, J. Non Cryst. Solids 45 (1981) p.397.

[32] S. N. Taraskin and S. R. Elliott, Phys. Rev. B 61 (2000) p.12031.

[33] S. N. Taraskin and S. R. Elliott, Phys. Rev. B 55 (1997) p.117.

[34] B. Hehlen, E. Courtens, R. Vacher, A. Yamanaka, M. Kataoka and K. Inoue, Phys. Rev. Lett. 84 (2000) p.5355.

10

(4)

(1)

(1)

(5)

(

.

4

(3)

.



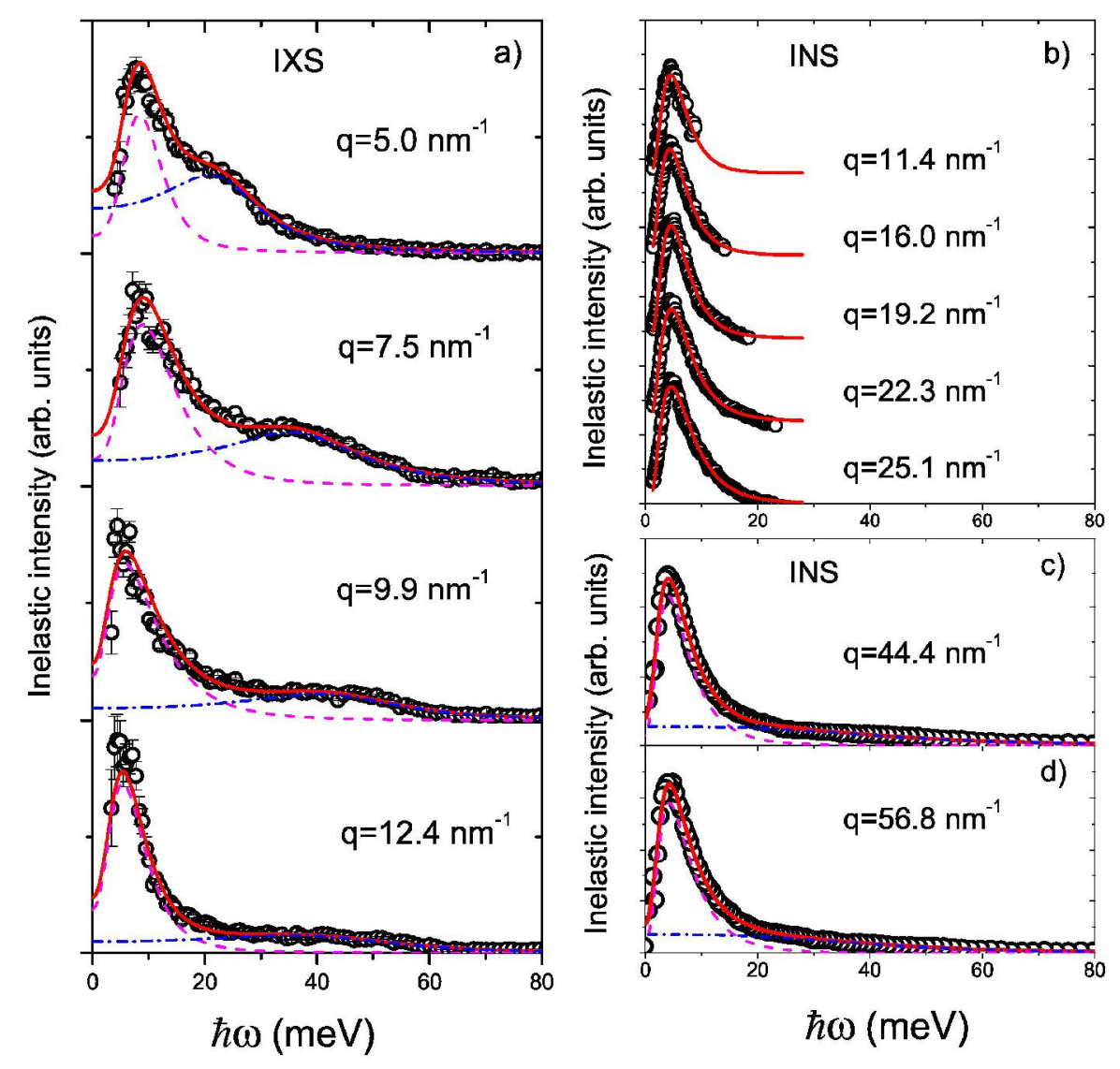

$126 \times 127 \mathrm{~mm}(600 \times 600 \mathrm{DPI})$ 


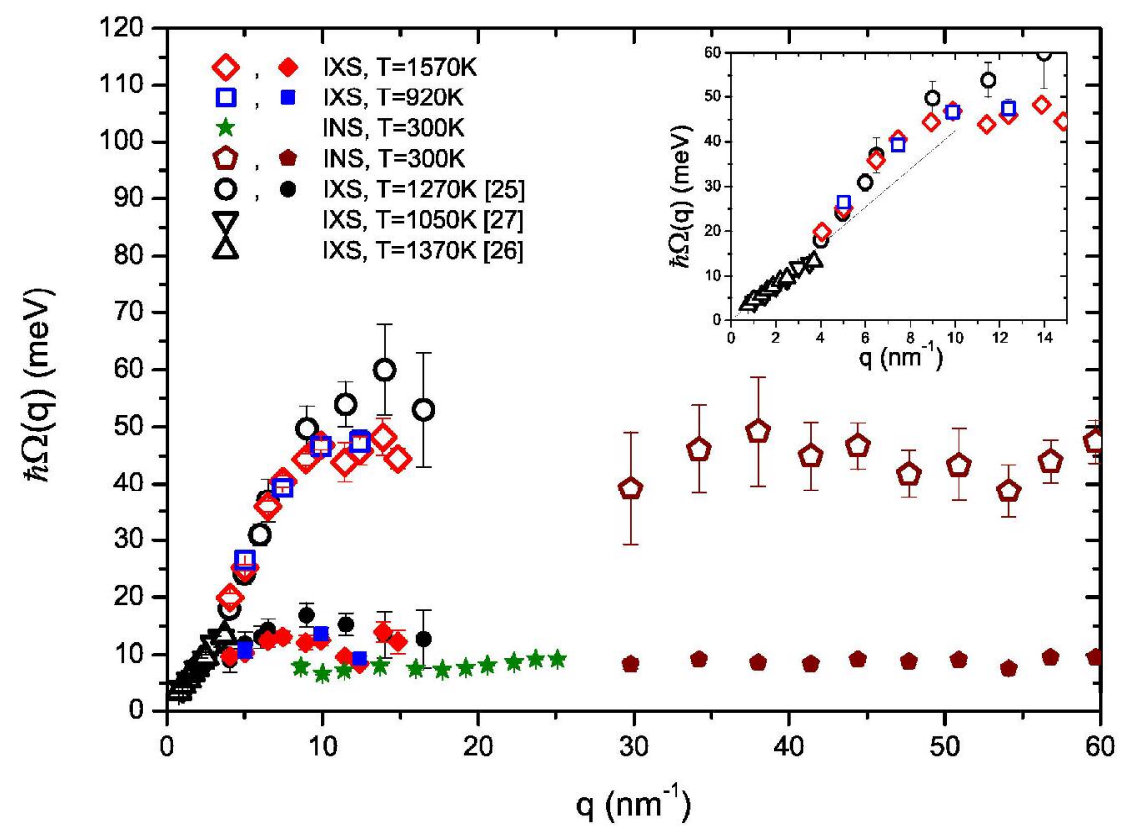

$109 \times 84 \mathrm{~mm}(600 \times 600 \mathrm{DPI})$ 

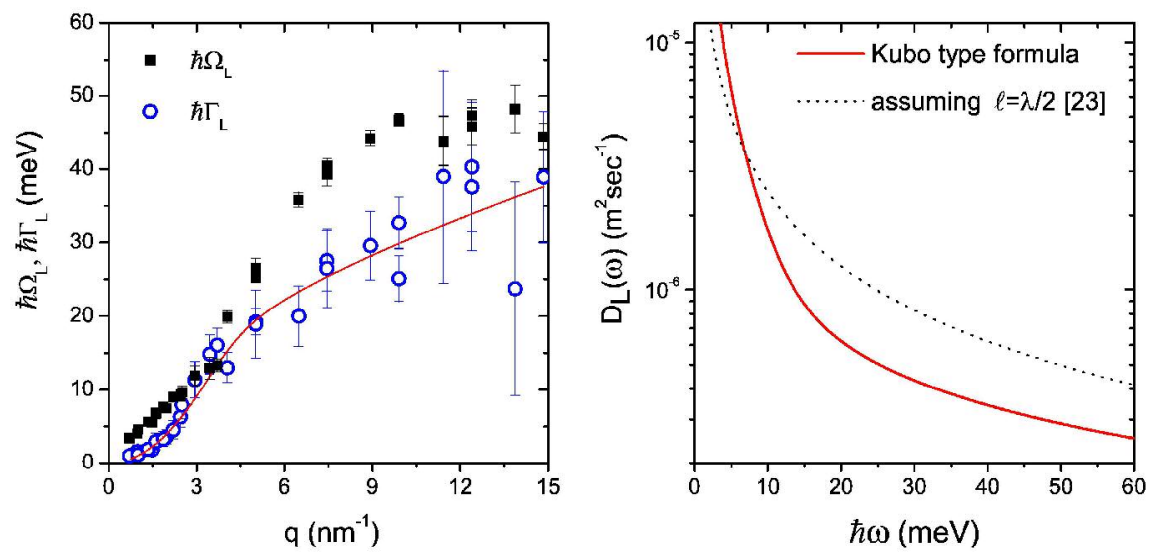

$170 \times 92 \mathrm{~mm}(600 \times 600 \mathrm{DPI})$ 


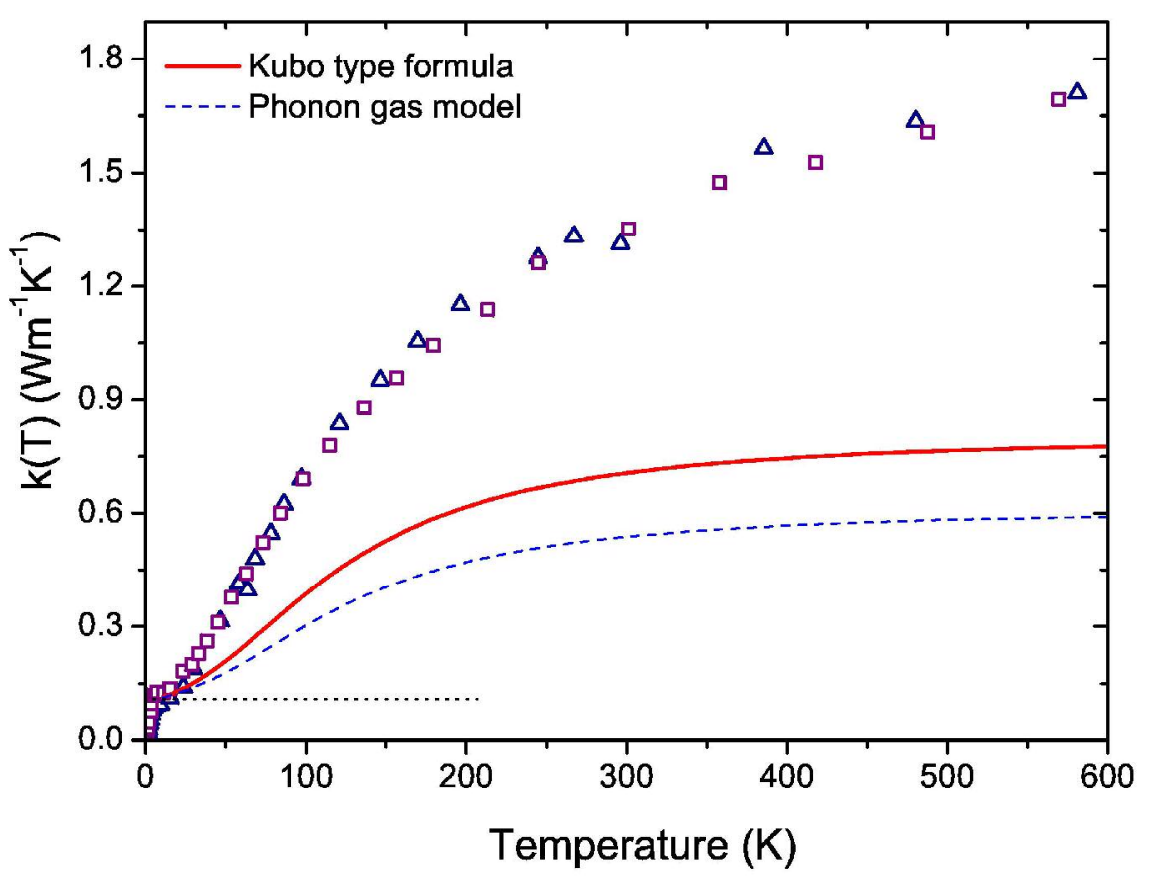

$113 \times 91 \mathrm{~mm}(600 \times 600 \mathrm{DPI})$ 